PROCEEDINGS OF THE

AMERICAN MATHEMATICAL SOCIETY

Volume 132, Number 8, Pages 2457-2460

S 0002-9939(04)07351-4

Article electronically published on March 24, 2004

\title{
A BRIEF REMARK ON VAN DER WAERDEN SPACES
}

\author{
ALBIN L. JONES
}

(Communicated by Alan Dow)

\begin{abstract}
We demonstrate that Martin's axiom for $\sigma$-centered notions of forcing implies the existence of a van der Waerden space that is not a Hindman space. Our proof is an adaptation of the one given by M. Kojman and S. Shelah that such a space exists if one assumes the continuum hypothesis to be true.
\end{abstract}

For the most part, we use standard set-theoretic language and notation, as presented in 4 and 8. All topological spaces are assumed to be Hausdorff.

An infinite set $A \in[\omega]^{\omega}$ is an AP-set if it includes arbitrarily long finite arithmetic progressions. It is an IP-set if there is an infinite set $B \in[\omega]^{\omega}$ for which $F S(B)=\left\{\sum F: \emptyset \neq F \in[B]^{<\omega}\right\}$, the collection of nonempty finite sums of $B$, is contained in $A$. Note that $\omega$ is both an AP-set and an IP-set.

Theorem 1 (van der Waerden, [9]). If an AP-set is partitioned into finitely many classes, then one of the classes must also be an AP-set.

Theorem 2 (Hindman, [3]). If an IP-set is partitioned into finitely many classes, then one of the classes must also be an IP-set.

Let $X$ be a topological space. Then $X$ is a van der Waerden space if every sequence $\left\{x_{n}: n<\omega\right\}$ in $X$ has a convergent subsequence $\left\{x_{n}: n \in A\right\}$ where $A$ is an AP-set. A sequence $\left\{x_{n}: n \in F S(B)\right\}$ in $X$ is IP-convergent to $x \in X$ if for every open neighborhood $U$ of $x$ there is an $m<\omega$ so that

$$
\left\{x_{m}: m \in F S(B \backslash m)\right\} \subseteq U .
$$

Then $X$ is a Hindman space if every sequence $\left\{x_{n}: n<\omega\right\}$ in $X$ has an IPconvergent subsequence $\left\{x_{n}: x \in F S(B)\right\}$ for some infinite $B \in[\omega]^{\omega}$. The notion of IP-convergence was introduced by Furstenberg and Weiss in [2], while van der Waerden and Hindman spaces were first explored by Kojman in [7] and [5], respectively.

Theorems 1 1and 2 together imply that all finite spaces are both van der Waerden and Hindman. In [7] and [5], Kojman proved that the classes of van der Waerden and Hindman spaces are each closed under finite products and properly contained in the class of sequentially compact spaces, and that any space in which the closure

Received by the editors March 13, 2003 and, in revised form, April 30, 2003.

2000 Mathematics Subject Classification. Primary 03E50, 05C55, 54F65, 11 P99.

Key words and phrases. General topology, continuum hypothesis, Martin's axiom, van der Waerden space, Hindman space, arithmetic sequence, convergent sequence.

We would like to thank both the University of Kansas for its support of this research and the anonymous referee for his helpful comments and suggestions. 
of any countable set is both compact and first countable belongs to both classes as well. These results certainly suggest the question of whether the two classes of spaces coincide, a question that Kojman and Shelah partially answered in [6].

An epiphany is a maximal almost disjoint family $\mathcal{M} \subseteq[\omega]^{\omega}$ such that each member of $\mathcal{M}$ is not an IP-set and such that for each AP-set $A$ and each finite-toone function $f: A \rightarrow \omega$ there is an AP-set $B \subseteq A$ so that $f[B]$ is contained in some element of $\mathcal{M}$.

Theorem 3 (Kojman and Shelah, [6]). If there is an epiphany, then there is a (compact) van der Waerden space that is not a Hindman space.

In [6], Kojman and Shelah proved that the continuum hypothesis implied the existence of an epiphany, and thus the existence of a space that is van der Waerden but not Hindman. Our goal is to prove that this same conclusion holds whenever Martin's axiom is true.

Proposition 4. Martin's axiom implies that there is an epiphany.

Proof. Let $\left\{\left(A_{\alpha}, f_{\alpha}\right): \omega \leq \alpha<\mathfrak{c}\right\}$ be an enumeration of all AP-sets $A_{\alpha}$ and finiteto-one functions $f_{\alpha}: A_{\alpha} \rightarrow \omega$. We will construct $\mathcal{M}=\left\{M_{\alpha}: \alpha<\mathfrak{c}\right\}$ by recursion on $\alpha<\mathfrak{c}$, and begin the construction by letting $\left\{M_{n}: n<\omega\right\}$ be any pairwise disjoint collection of non-IP-sets.

Assuming only that $\left\{M_{\beta}: \beta<\alpha\right\}$ have been successfully articulated, we must define $M_{\alpha}$. Consider $A_{\alpha}$ and $f_{\alpha}: A_{\alpha} \rightarrow \omega$. If there is $\beta<\alpha$ so that $f_{\alpha}^{-1}\left[M_{\beta}\right]$ is an AP-set, then just let $M_{\alpha}=M_{\beta}$.

Otherwise, we will use Martin's axiom to produce an AP-set $B \subseteq A_{\alpha}$ for which $M_{\alpha}=f_{\alpha}[B]$ is a non-IP-set that is almost disjoint from each previously defined $M_{\beta}$.

Define a notion of forcing $\mathbb{P}$ as follows. Conditions of $\mathbb{P}$ are pairs $p=\left(B_{p}, F_{p}\right)$ with $B_{p} \in\left[A_{\alpha}\right]^{<\omega}$ a finite approximation to $B$ and $F_{p} \in[\alpha]^{<\omega}$ a finite set of "working" indices. For $p, q \in \mathbb{P}$, put $q \Vdash p$ if and only if $B_{q} \supseteq B_{p}, F_{q} \supseteq F_{p}$,

$$
B_{q} \backslash B_{p} \subseteq A_{\alpha} \backslash f_{\alpha}^{-1}\left[\bigcup\left\{M_{\beta}: \beta \in F_{p}\right\}\right],
$$

each element of $f_{\alpha}\left[B_{q} \backslash B_{p}\right]$ is at least $2 \cdot \max f_{\alpha}\left[B_{p}\right]$, and the absolute difference between each distinct pair of elements of $f_{\alpha}\left[B_{q} \backslash B_{p}\right]$ is at least $\max f_{\alpha}\left[B_{p}\right]$. The first three conditions will ensure that $B$ is an AP-set and that $f_{\alpha}[B]$ is almost disjoint from each element of $\left\{M_{\beta}: \beta<\alpha\right\}$, while the last two conditions will guarantee that $f_{\alpha}[B]$ is not an IP-set.

Note that $A_{\alpha} \backslash f_{\alpha}^{-1}\left[\bigcup\left\{M_{\beta}: \beta \in F\right\}\right]$ is an AP-set for all finite $F \subseteq \alpha$.

Claim 4.1. For each $n<\omega$, the set $\mathcal{A}_{n}$ of all $p \in \mathbb{P}$ for which $B_{p}$ includes an $n$-term arithmetic progression is dense in $\mathbb{P}$.

Proof. Let $p=\left(B_{p}, F_{p}\right)$ be an arbitrary element of $\mathbb{P}$. As we noted above, $A=$ $A_{\alpha} \backslash f_{\alpha}^{-1}\left[\bigcup\left\{M_{\beta}: \beta \in F_{p}\right\}\right]$ is an AP-set. Let $m=\max f_{\alpha}\left[B_{p}\right]$. For each $r<m$ let $E_{r}=\{k \cdot m+r: 2 \leq k<\omega\}$. Note that $\left\{A \cap f_{\alpha}^{-1}\left[E_{r}\right]: r<m\right\}$ is a partition of all but finitely many elements of $A$ into finitely many classes. By Theorem 1 , there must be $r<m$ so that $A \cap f_{\alpha}^{-1}\left[E_{r}\right]$ is an AP-set. Choose an $n$-term arithmetic progression $C \subseteq A \cap f_{\alpha}^{-1}\left[E_{r}\right]$. Put $q=\left(B_{p} \cup C, F_{p}\right)$. Clearly, $q \Vdash p$ and $B_{q}$ includes an $n$-term arithmetic progression. Thus, $\mathcal{A}_{n}$ is dense in $\mathbb{P}$. 
Claim 4.2. For each $\beta<\alpha$, the set $\mathcal{B}_{\beta}$ of all $p \in \mathbb{P}$ for which $\beta \in F_{p}$ is dense in $\mathbb{P}$.

Proof. Let $p=\left(B_{p}, F_{p}\right)$ be an arbitrary element of $\mathbb{P}$. Let $q=\left(B_{p}, F_{p} \cup\{\beta\}\right)$. Clearly, $q \Vdash p$. Thus, $\mathcal{B}_{\beta}$ is dense in $\mathbb{P}$.

Claim 4.3. $\mathbb{P}$ is $\sigma$-centered.

Proof. Clearly, any $p, q \in \mathbb{P}$ with $B_{p}=B_{q}$ are compatible.

We may therefore use Martin's axiom to find a filter $G \subseteq \mathbb{P}$ that simultaneously meets each $\mathcal{A}_{n}$ for $n<\omega$ and each $\mathcal{B}_{\beta}$ for $\beta<\alpha$. Once such a filter has been found, let $B=\bigcup\left\{B_{p}: p \in G\right\}$. That $G$ meets $\mathcal{A}_{n}$ for each $n<\omega$ ensures that $B$ is an AP-set, and that $G$ meets $\mathcal{B}_{\beta}$ for each $\beta<\alpha$ ensures that $f_{\alpha}[B]$ is almost disjoint from each $M_{\beta}$ for $\beta<\alpha$. It is easily verified that $\lim _{n \rightarrow \infty}\left(b_{n+1}-b_{n}\right)=\infty$, where $b_{n}$ is the $n$th element of $f_{\alpha}[B]$ in increasing order for each $n<\omega$. This is enough to ensure that $f_{\alpha}[B]$ is not an IP-set. Let $M_{\alpha}=f_{\alpha}[B]$.

Let $\mathcal{M}=\left\{M_{\alpha}: \alpha<\mathfrak{c}\right\}$. We need only verify that $\mathcal{M}$ is an epiphany.

Clearly, each element of $\mathcal{M}$ is not an IP-set.

Suppose that $A$ is an AP-set and $f: A \rightarrow \omega$ is a finite-to-one function. Then there must be an infinite $\alpha<\mathfrak{c}$ with $A=A_{\alpha}$ and $f=f_{\alpha}$. If there is $\beta<\alpha$ so that $f^{-1}\left[M_{\beta}\right]$ is an AP-set, then let $B=f^{-1}\left[M_{\beta}\right]$. Otherwise, let $B=f^{-1}\left[M_{\alpha}\right]$. Either way, $B \subseteq A$ is an AP-set for which there is $M \in \mathcal{M}$ with $f[B] \subseteq M$.

To see that $\mathcal{M}$ is a maximal almost disjoint family, suppose $N \in[\omega]^{\omega}$. Let $f: \omega \rightarrow N$ be the increasing enumeration of $N$. There are an AP-set $B$ and $M \in \mathcal{M}$ with $f[B] \subseteq M$. Clearly then, $N \cap M$ is infinite.

The proof actually shows that the same conclusion follows from Martin's axiom restricted to $\sigma$-centered forcings. In [1, M. Bell proved that this weakened version of Martin's axiom is equivalent to the assertion that $\mathfrak{p}=\mathfrak{c}$.

Corollary 5. Assume that $\mathfrak{p}=\mathfrak{c}$. Then there is a van der Waerden space that is not a Hindman space.

Proof. This now follows directly from Theorem 3 and our proof of Proposition 4

Question 1. Is it consistent that there is a Hindman space that is not a van der Waerden space?

Question 2. Is it consistent that all Hindman spaces are van der Waerden spaces and vice versa?

\section{REFERENCES}

[1] Murray G. Bell, On the combinatorial principle P(c), Fund. Math. 114 (1981), no. 2, 149-157. MR 83e:03077

[2] H. Furstenberg and B. Weiss, Topological dynamics and combinatorial number theory, J. Analyse Math. 34 (1978), 61-85 (1979). MR 80g:05009

[3] Neil Hindman, Finite sums from sequences within cells of a partition of $N$, J. Combinatorial Theory Ser. A 17 (1974), 1-11. MR 50:2067

[4] T. Jech, Set theory, Pure and Applied Mathematics, Academic Press [Harcourt Brace Jovanovich Publishers], New York, 1978. MR 80a:03062

[5] M. Kojman, Hindman spaces, Proc. Amer. Math. Soc. 130 (2002), no. 6, 1597-1602. MR 2003c:54068

[6] M. Kojman and S. Shelah, Van der Waerden spaces and Hindman spaces are not the same, Proc. Amer. Math. Soc. 131 (2003), no. 5, 1619-1622. MR 2004c:54003 
[7] Menachem Kojman, Van der Waerden spaces, Proc. Amer. Math. Soc. 130 (2002), no. 3, 631-635 (electronic). MR 2002i:54018

[8] K. Kunen, Set theory: An introduction to independence proofs, reprint of the 1980 original, North-Holland Publishing Co., Amsterdam, 1983. MR 85e:03003

[9] B. L. van der Waerden, Beweis eine Baudetschen Vermutung, Nieuw Arch. Wisk. 15 (1927), $212-216$.

Department of Mathematics, University of Kansas, Lawrence, Kansas 66045-2142

E-mail address: alj@math.ku.edu

$U R L:$ http://www.math.ku.edu/ alj 